\title{
Context Adaptive Self-configuration System
}

\author{
Seunghwa Lee and Eunseok Lee \\ School of Information and Communication Engineering, Sungkyunkwan University, \\ 300 Chunchun jangahn Suwon, 440-746, Korea \\ \{jbmania, eslee\}@.selab.skku.ac.kr
}

\begin{abstract}
This paper suggests a context adaptive self-configuration system, in which the system itself is aware of its available resources, and executes a configuration suitable for each system and the characteristics of each user. A great deal of time and effort is required to manually manage computing systems, which are becoming increasingly larger and complicated. Many studies have dealt with these problems but most unfortunately have focused on 'automation', which do not reflect the system specifications that differ between systems. Therefore, this paper proposes an adaptive self-configuration system that collects diverse context information for a user and its system, reflects it to an auto-response file and executes the detail parameter setting automatically. A prototype was developed to evaluate the system and compare the results with the conventional methods of manual configuration and MS-IBM systems. The results confirmed the effectiveness of the proposed system.
\end{abstract}

\section{Introduction}

Recently, the rapid development of IT has contributed to various devices with increasing performances. As the devices to be administered are increasing in number and complication, they impose a heavier burden on system management [1]. For examine, a company's computing system networking hundreds of hosts may require a great deal of time, money and manual effort if they have to be manually and individually installed and updated. In order to solve it, studies such as a centralized integration management of distributed resources, and unattended installation technology to reduce the workload and periodic automatic update are being applied by various institutions [2][3]. However, these studies focused mainly on 'automation', which simply replaces the tasks manually processed. Therefore, these studies provide only a uniform service that does not reflect unique device's characteristics or the user preferences. This paper proposes a context adaptive self-configuration system, which is aware of its available resources, and executes the suitable configuration on each situation utilizing these contexts with the user's information.

The paper is presented as follows: Section 2 describes the characteristics, structure and behavior process of the proposed system; and Section 3 gives a conclusion as well as a simple description of the system evaluation through its implementation and experiments. 


\section{Proposed System}

\subsection{System Overview}

The word 'configuration' in this paper means newly install components, such as an OS or software, etc., that are to be administered and updated them for version management or defects healing.

For installation, the proposed system need be installed so that components to be contained reflect the existing users' preference and the system resource status. The basic configuration automatically meets these requirements through a customized auto-response file and a parameter-setting file. In addition, for updating, it monitors the frequency of component use, determines the priority, and enables works to be efficiently processed in case the remaining storage of a system or the updating time is insufficient. Currently, copying files is processed by a peer-to-peer transmission method to supplement the several disadvantages of the existing centralized distribution method and to efficiently update the files.

\subsection{System Structure}

Fig. 1 shows the overall structure of the proposed system, and the detail modules are as follows:

Component Agent: this is installed in a managed device to monitor the changes in resources, to check the parameter setting information of the user's applications along with the frequency of use, and to periodically transmit the this information to a selfconfiguration system. In addition, it also receives packages or the configuration files necessary for auto installation from the self-configuration system or a host located in the same zone. Upon installation, it observes the user's behavior, and transmits the observed information to the Context Synthesizer.

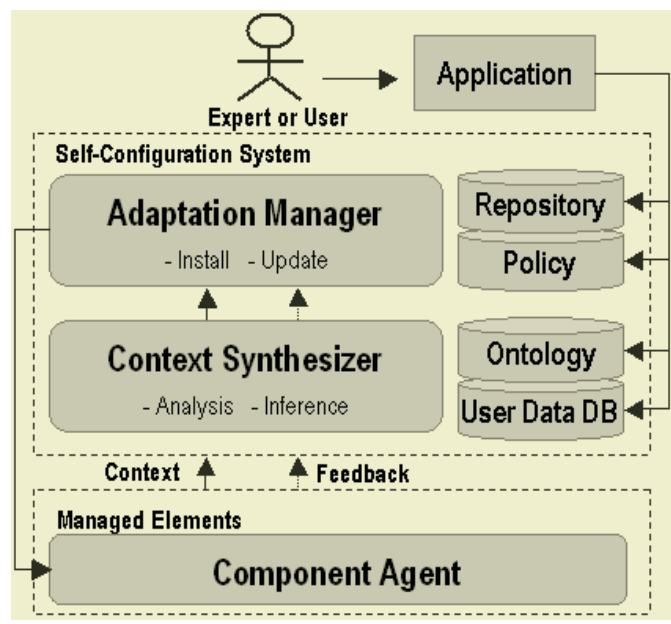

Fig. 1. Architecture of the Proposed system

- Context Synthesizer: Gathers the context provided by the Component Agent and stores the basic user data, the application preferences and usage frequencies in the User Data DB. The Context Synthesizer analyzes and deducts the current status using the stored user data and Ontology (various 
expressions on the preference data of the applications) and relays the results to the Adaptation Manager.

- Adaptation Manager: Decides and performs the appropriate tasks based on policies that were pre-defined through the data provided by the Context Synthesizer. The Adaptation Manager installs the components stored in the Repository or performs the various configuration tasks such as updates. An installation image on the user data and the customized 'auto-response files' for installation are also automatically created.

The system administrator or the user can adjust and manage these components, policies, Ontology, and the user data to be stored in the Repository. The proposed system can also communicate with other external Configuration Servers and share data.

\subsection{System Behavior}

\section{1) Installation}

The system installs the components by reflecting the existing user's preference and system resource information, and automatically executes the basic configuration through a customized auto-response file and an auto configuration file. In addition, in case a user's resource is insufficient, it automatically selects the 'minimum installation' option for the auto-response file, creating the script. Currently, such a process is notify to the user so that the final decision may be made by the user whilst an agent observes and learns the user's activities, which are reflected to determine the subsequent activities. The major workflow is as follows.

Step 1. Component Agent: monitors the system resource information and configuration information, creating a profile and transmitting it to a context synthesizer, for any changes.

Step 2. Context Synthesizer: saves the received data to the User Data DB, on which the present situation is analyzed.

Step 3. In case a user requests an installation or an administrator needs to distribute it in a lump, it retrieves the user data (preference) saved in the User Data DB, after authenticating the user.

Step 4. Adaptation Manager: creates and distributes the auto-response file and the detail parameter setting file, based on the retrieved user data. (ex. if (resource = insufficient) then checks the "minimum installation' option of the auto-response file)

Step 5. Component Agent: installs any received file, monitors the user's activities and executes the feedback.

Step 6. Modifies and learns the User Preference, Policy.

Fig. 2. Pseudo Code for the Install Phase

\section{2) Update}

The update priority is determined by monitoring the frequency of the components, through which the system facilitates the various tasks efficiently if the remaining storage or the time required for updating is insufficient. Reflecting the Astrolabe [4] structure, each host organizes the components into zones, and these zones are organized hierarchically. A representative host of each zone collects a list of files on 
the individual hosts while a host requiring the file requests the representative host about the location of a near host holding the file, and receives it in peer-to-peer transmission method. From this, it can reduce the load of the centralized server, be resistant to various troubles and quickly copy files. The update files copied within the zone are checked for consistency through a size comparison.

Step 1. Component Agent: monitors the frequency of each application by the users, transmits it to the context synthesizer periodically and sends its own update file list to the representative host in the same zone. In addition, the representative host's collected file lists are gathered at the higher level.

Step 2. Context Synthesizer: saves the received data into the User Data DB, on which the user's preference is present.

Step 3. For any update request (update request by a user or update request at a lump by an administrator), it authenticates the user and retrieves the user's data saved in User Data DB.

Step 4. Adaptation Manager: determines the update priority according to the retrieved user data.

Step 5. The system requests the representative host in the same zone, the location of a host holding the file, catches hold of it and finally copies the file. (if the file needed does not exist at the representative host, then go to the level and submit the request to the higher level host).

Fig. 3. Pseudo Code for the Update Phase

\section{Conclusion}

This study proposed a prototype system for testing the basic functions and validating a system by comparing for the task time for the existing research. From the results, it could be concluded that proposed system features a shortened configuration work time than the existing systems (shortening the basic parameter setting time) and an increased the user satisfaction and usability. Meanwhile, it is expected that the proposed system might be useful in the Ubiquitous Computing era where a user utilizes multiple computing devices and provides them with a more convenient computing environment as a result of the reduced system management.

\section{References}

1. Paul Horn, "Autonomic Computing: IBM's Perspective on the State of Information Technology”, IBM White paper, Oct.2001

2. http://www-306.ibm.com/software/tivoli

3. http://www.microsoft.com/technet/prodtechnol/winxppro/deploy/default.mspx

4. Robbert van Renesse, Kenneth Birman and Werner Vogels, "Astrolabe: A Robust and Scalable Technology for Distributed System Monitoring, Management, and Data Mining", ACM Transactions on Computer Systems, Vol.21, No.2, pp.164-206, May 2003

5. John Keeney, Vinny Cahill, "Chisel: A Policy-Driven, Context-Aware, Dynamic Adaptation Framework", In Proceedings of the Fourth IEEE International Workshop on Policies for Distributed Systems and Networks, Jun.2003

6. Anand Ranganathan, Roy H. Campbell, "A Middleware for Context-Aware Agents in Ubiquitous Computing Environments”, In ACM/IFIP/USENIX International Middleware Conference 2004, Jun.2004

7. Richard S. Sutton, Andrew G. Barto, 'Reinforcement Learning: An Introduction (Adaptive Computation and Machine Learning)', The MIT Press, Mar.1998 